\title{
Modeling the Dynamics of Endemic Malaria Transmission with the Effects of Control Measure
}

\author{
Dereje Gutema Edossa, Alemu Geleta Wedajo, Purnachandra Rao Koya
}

Department of Mathematics, Wollega University, Nekemte, Ethiopia

Email address:

derejegutema8@gmail.com (D. G. Edossa), alemugeleta@gmail.com (A. G. Wedajo), drkpraophd@gmail.com (P. R. Koya)

\section{To cite this article:}

Dereje Gutema Edossa, Alemu Geleta Wedajo, Purnachandra Rao Koya. Modeling the Dynamics of Endemic Malaria Transmission with the Effects of Control Measure. American Journal of Applied Mathematics. Vol. 8, No. 3, 2020, pp. 158-170. doi: 10.11648/j.ajam.20200803.17

Received: December 27, 2019; Accepted: June 3, 2020; Published: June 17, 2020

\begin{abstract}
Malaria is an infectious disease caused by Plasmodium parasite and is transmitted among humans through bites of female Anopheles mosquitoes. It is estimated 216 million people suffered from malaria in 2016, with over 400,000 deaths mainly in sub-Saharan Africa. A number of control measures have been put in place: most importantly the insecticide treated net (ITN) and indoor residual sprayings (IRS) of insecticide. Currently, the emergence and spread of resistance in mosquito populations against insecticides is the most common and widely spread .It is also poses a key obstacle to malaria control as well as jeopardizing the effects of the most efficient malaria control interventions. A mathematical model that incorporates the evolution of insecticide resistance and its impact on endemic malaria transmission i.e., effects of indoor residual sprayings (IRS) on the insecticide resistant and sensitive malaria vector strains as a control strategy is incorporated and analyzed. The object of the study is to understand qualitatively the factor that have more influence for the emergence and spread of resistance of malaria vectors against IRS and their impacts on the efficacy of IRS. Based on a Ross-Macdonald derivation of malaria model the effective reproduction number $R_{e}$ isused to assess the effects of IRS in the qualitative analysis of the model. The existence and stability of the disease-free and endemic equilibria of the model are studied. It is established that the malaria can be brought under control as long as $\Re_{e}$ is kept below the threshold value. Numerical simulations studies are conducted so as to determine the role played by key parameters of the model. The public health implications of the results include: (i) every effort should be taken to minimize the evolution of insecticide resistance due to malaria control interventions failure and (ii) at least a combination of two types of different control measures and followed by rotation of intervention strategies could be more realistic to minimize the number of resistant malaria vector strains and essential in reducing the malaria burden in the community.
\end{abstract}

Keywords: Endemic Malaria, Infectious, Insecticide, Emergence, Resistance, Modeling, Mosquito

\section{Introduction}

Malaria is an infectious disease caused by the Plasmodium parasite and is transmitted among humans through bites of female Anopheles mosquitoes. Also, it is transmitted more infrequently by blood transfusion i.e., needle sharing, surgery and births [1]. Among all the diseases those can be transmit by mosquitoes, malaria has been and still remains the one having the greatest health and socioeconomic impact, from the ancient Egypt to present time [2,3].

Nearly half of the world's population is at risk of Malaria disease. In 2016, about 216 million people suffered from malaria and among them over 0.4 million people lost their lives. The intensity is still more mainly in sub-Saharan Africa [4].
The main symptoms of malaria include fatigue, chill, headache, abdominal and back pain, diarrhea, sometimes vomiting, and fever.

In recent years global efforts have been made to control and eliminate malaria. This effort has lead to a significant reduction in malaria cases and mortality at rates of $66 \%$ and $42 \%$ in Africa respectively.

Various techniques have been followed to prevent and cure the Malaria disease. Preventive techniques of Malaria disease include: the use of insecticide-treated nets ITNs, long-lasting insecticidal nets LLINs and indoor residual sprayings IRS [5]. Curing techniques of Malaria disease include: Early diagnosis, improved drug therapies and better health infrastructure.

The 'insecticide resistance' is defined as the ability of an 
insect to withstand from the effects of an IRS [7]. Repeated exposure to insecticides may make the insects more rapidly resistant to or are less sensitive to that. These individual survivors could then pass the resistance mechanism to the successive generations resulting in the production of more resistant insect populations [8].

Currently, the resistance against insecticides in mosquito populations is emerging and spreading. This face is disturbing the effectiveness of the most efficient malaria control interventions [6].

Insecticide resistant malaria vector strains limits the effectiveness of control and intervention strategies. This also resulted in higher: malaria morbidity and mortality, increased cost of malaria disease management, increased burden on the health care facilities, and increased relative malaria incidence and parasite infection prevalence within individuals.

Mathematical modeling of malaria has been flourishing since the days of Ronald Ross, in 1911, who was awarded a Nobel Prize for his contributions. A simple SI model having two compartments namely susceptible and infected is developed. This model has a basis on the assumption that at any time, the total human population can be divided into some distinct compartments. The so called mathematical model is used to show that bringing mosquito population below a certain threshold is sufficient to eliminate malaria. This threshold naturally depends on biological factors such as the biting rate and vectors capacity [9].

Macdonald G. has developed a model for estimating the infection and recovery rates. This model assumes that the amount of infective material to which a population is exposed remains unchanged. It is shown that the reduction of the number of mosquitoes is an inefficient control strategy since that had a little effect on the dynamics of malaria in areas of strong transmission [9, 10].

The Ross - Macdonald developed a reformulated model an interaction between infected human hosts and infected mosquito vectors that identifies mosquito vector longevity as the single most important variable in the force of transmission, and combined Ross's model with epidemiological and entomological file data Since then, study developed on mosquito-borne pathogen transmission and designed strategies for mosquito-borne disease prevention $[11,12,13,14,15,16,17]$.

The models developed by Aronhave considered that acquired immunity to malaria depends on exposure i.e. the immunity is boosted by additional infections $[18,19]$.

Recently, Tumwiine, Mugisha and Luboobi have developed a compartmental model to formulate the spread of malaria, with susceptible - Infected - Recovered Susceptible SIRS pattern for human and Susceptible Infected SI pattern for mosquitoes [20, 21].

Yang, Wei and Li have developed a model consisting of SIR compartments for human and SI compartments for vector populations respectively. The concept of reproduction number $R_{0}$ is used. Further, the existence and stability of disease-free and an endemic equilibria are proposed [22].

Fekadu Tadege Kobe and Purnachandra Rao Koya have developed a model and shown that the spread of malaria disease can be controlled using effective intervention strategies [23].

Mathematical model is a valuable tool in the study of the dynamics of diseases. It provides the abilities of understanding and predictions of epidemiological patterns and dynamical nature of diseases.

Ross - Macdonald model focused on only one factor that mosquito vector longevity as the single most important variable. This variable is used in the force of transmission of the disease and strategies for mosquito-borne disease prevention.

Some other important issued missed to consider are:(i) complex dynamics of the host-vector interactions (ii) evolution of insecticide resistance (iii) factors that influence the resistance of malaria vectors against insecticides and (iv) the efficacy of control measures.

In the present model few of the listed factors have been incorporated and thus extended the Ross - Macdonald model. Here, the human population is compartmentalized as susceptible-infective-immune SIR and mosquito population is compartmentalized as susceptible-infective SI. Further, the infected mosquito population is divided into two classes: (i) insecticide sensitive and (ii) insecticide resistant. Also, considered that the infection with the insecticide sensitive strain will give rise the insecticide resistant strain in the event of indoor residual spray IRS fails to kill mosquito vectors.

\section{Model Formulation and Analysis}

\subsection{Model Formulation}

In this section, an improved mathematical model for the transmission and spread of malaria disease between two interacting populations of humans or the host and mosquitoes or the vector is developed. This model is an extension of that of Ross - Macdonald.

This model compartmentalizes the total human population denoted by $N_{h}(t)$ at time $t$ into three classes: susceptible $S_{h}(t)$, infected $I_{h}(t)$, and immune $R_{h}(t)$ classes. Hence, the total human population is given by $N_{h}(t)=S_{h}(t)+I_{h}(t)+R_{h}(t)$.

Similarly, the total mosquito population is divided into two classes: Susceptible $S_{v}(t)$ and infectious $I_{v}(t)$. Further, the infected mosquito population classified as: insecticide sensitive malaria vectors $I_{v s}$ and insecticide resistant malaria vectors $I_{v r}$. Here, the subscripts $s$ and $r$ are added to the vector variables in order to specify the sensitive and resistant strains respectively. Thus, the total mosquito population at any time $t$ is denoted and given by $N_{v}(t)=S_{v}(t)+I_{v s}(t)+I_{v r}(t)$.

The mosquitoes do not have any recovered class since the infected mosquitoes remain infectious for whole life. The present model ignores super-infections for mosquitocompartments and the latent periods of the disease for both human and mosquitocompartments.

The assumptions of the present model include the following: Individuals are recruited into the susceptible class with a rate of $\Lambda_{h}$ due to births and/or immigrations. Both insecticide sensitive and infected insecticide resistant malaria 
vectorsattack susceptible human with the probabilities of $\beta_{h}$ and $\left(1-\beta_{h}\right)$ respectively [24].

In the process, the parasite injects sporozoites into the blood and move to the infectious compartment $I_{h}(t)$. Infectious humans recover due to immunity resistance with a rate of $\gamma$. Since immunity against malaria infection is temporary, it is lost at a constant rate $\theta$ and the immune humans become susceptible again. All humans are subjected to a non-disease related per capita natural death rate $\mu_{h}$. Additionally, disease related deaths occur with a constantrate of $\delta_{h}$ in the infective class.

In mosquito population the birth rate is considered to be a constant $\Lambda_{v}$ per capita. Thesusceptible mosquito becomes infected with a probability $\beta_{v}$ from biting infectious human $I_{h}(t)$. Let a fraction of $\rho$ susceptible mosquitoes moveto insecticide sensitive malaria vector strains and the remaining fraction $(1-\rho)$ moveto insecticide resistant malaria vector strains, where $0 \leq \rho \leq 1$.

The effect of IRS is incorporated solely for the death of the mosquito populations. That is, both the susceptible female mosquitoes $S_{v}$ and insecticide sensitive malaria vector strains $I_{v s}$ are expected to be killed by IRS. The parameter $\alpha$ is the rate of removal of mosquitoes from different classes associated with IRS. The values of IRSapplied per day ranges from 0 to 1 . The amount of IRS used is incremented by a constant so as to account for a wide range of efficacies and compliancesapplicable.

The evolution of insecticide resistancedue to improper use of IRSleading to the control intervention failure is also incorporated in the model. Thus, the rate at whichinsecticide sensitive malaria vector strains progress to theinsecticide resistant malaria vector strains class is considered to be $\varphi$. All mosquitoes are subjected to a non-diseasednatural death rate $\mu_{v}$ per capita. Additionally, disease related death rate is considered to be a constant $\delta_{v}$ in both the sensitive and resistant infective classes.

\subsection{Model Assumptions}

The formulation of the present model is guided by the following assumptions:

(i) The total sizes of both humans and mosquito populations are not constant.

(ii) The mosquito populations cannot be completely eliminated and thus there will be ongoing transitions of the disease.

(iii) Insecticideresistant malaria vector strains will affect both mosquito populations and the effectiveness of control intervention and hence maximizes the potential of disease transmission.

(iv) The emergency of insecticide resistant malaria vectorstrainisbased on quality (improper usage) and quantity (types) of control interventions.

(v) Spraying of IRS on the places where mosquitoes reproduce and on the home walls leads to the death of mosquito populations.

(vi) On recovery, humans will havetemporary immunity.

(vii) The number of insecticide resistant vectors will increase with time due to the failure of effectiveness of IRS on sensitive vectors.

(viii) The populations in compartmentsof both humans and vectors are non-negative and so areall the parameters involved in the model.

(ix) Malaria isactivein a population for a long period of time.

Table 1. Description of state variables.

\begin{tabular}{ll}
\hline State Variable & Description \\
\hline $\mathrm{S}_{\mathrm{h}}(\mathrm{t})$ & Number of Susceptible Humans \\
$\mathrm{I}_{\mathrm{h}}(\mathrm{t})$ & Number of Infected Humans \\
$\mathrm{R}_{\mathrm{h}}(\mathrm{t})$ & Number of Recovered Humans \\
$\mathrm{S}_{\mathrm{v}}(\mathrm{t})$ & Number of Susceptible Mosquitoes \\
$\mathrm{I}_{v s}(\mathrm{t})$ & Number of Infected insecticide sensitive Mosquitoes \\
$\mathrm{I}_{\mathrm{vr}}(\mathrm{t})$ & Number of Infected insecticide resistant Mosquitoes \\
\hline
\end{tabular}

Table 2. Description ofmodelparameters.

\begin{tabular}{ll}
\hline Parameter & Description \\
\hline$\Lambda_{\mathrm{h}}$ & Recruitment rate of Susceptible Humans \\
$\Lambda_{\mathrm{v}}$ & Recruitment rate of Susceptible Mosquitoes \\
$\mu_{\mathrm{h}}$ & Natural death rate of Humans \\
$\delta_{\mathrm{h}}$ & Disease-induced death rate of Humans \\
$\beta_{\mathrm{h}}$ & Contact rate of Infective Vector and Susceptible Humans \\
$\gamma$ & Recovery rate of Infective Humans \\
$\mu_{\mathrm{v}}$ & Natural death rate of Mosquitoes \\
$\delta_{\mathrm{v}}$ & Disease-induced death rate of Humans \\
$\theta$ & Rate of loss of immunity in Humans \\
$\beta_{\mathrm{v}}$ & Contact rate of Susceptible Mosquitoes and Infective \\
$\alpha$ & Humans \\
\hline
\end{tabular}

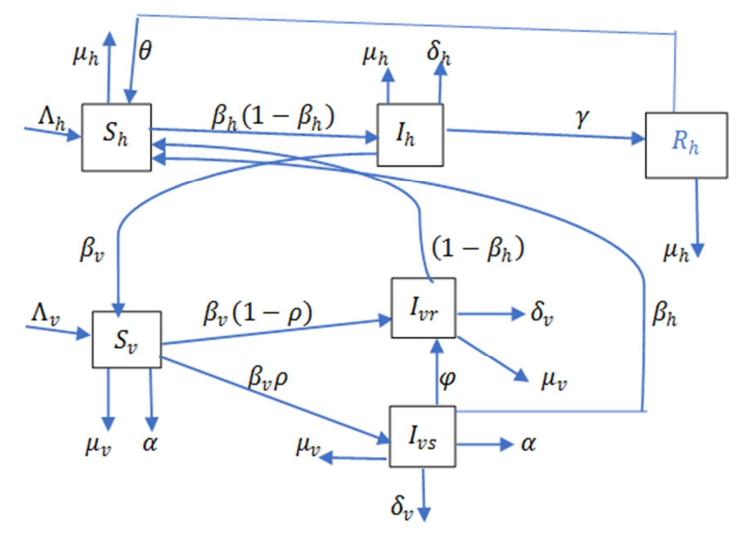

Figure 1. Flow of Malaria parasite between Humans and Mosquitoes.

Based on the model assumptions, description of model parameters and the state variables and the flow chart given in the Figure 1, the system of model equations can be developed as follows:

$$
\begin{array}{r}
d S_{h} / d t=\Lambda_{h}+\theta R_{h}-\left[\beta_{h}\left(1-\beta_{h}\right)\left(I_{v s}+I_{v s}\right) S_{h}\right] / N_{h}-\mu_{h} S_{h}(1) \\
d I_{h} / d t=\left[\beta_{h}\left(1-\beta_{h}\right)\left(I_{v s}+I_{v s}\right) S_{h}\right] / N_{h}-\left(\gamma+\mu_{h}+\delta_{h}\right) I_{h}(2) \\
d R_{h} / d t=\gamma I_{h}-\left(\mu_{h}+\theta\right) R_{h} \\
d S_{v} / d t=\Lambda_{v}-\left[\beta_{v} S_{v} I_{h}\right] / N_{v}-\left(\alpha+\mu_{v}\right) S_{v}(4) \\
d I_{v s} / d t=\left[[\rho(1-\varphi)] \beta_{v} S_{v} I_{h}\right] / N_{v}-\left(\alpha+\mu_{v}+\delta_{v}\right) I_{v s}(5) \\
d I_{v r} / d t=\left[(1-(1-\varphi) \rho) \beta_{v} S_{v} I_{h}\right] / N_{v}-\left(\mu_{v}+\delta_{v}\right) I_{v r}(6)
\end{array}
$$




$$
\begin{gathered}
d N_{h} / d t=\Lambda_{h}-\mu_{h} N_{h}-\delta_{h} I_{h} \\
d N_{v} / d t=\Lambda_{v}-\mu_{v} N_{v}-\alpha\left(S_{v}+I_{v s}\right)-\delta_{v}\left(I_{v} s-I_{v r}\right)
\end{gathered}
$$

With initial conditions

$$
\begin{aligned}
S_{h}(0)= & S_{0 h}, I(0)=I_{0 h}, R_{h}(0)=R_{0 h}, S_{v}(0)=S_{0 v}, N_{v}(0)= \\
& N_{0} v I_{v s}(0)=I_{0 v s}, I_{v r}(0)=I_{0 v r}, N_{h}(0)=N_{0 h},
\end{aligned}
$$

\subsection{Model Analysis}

\subsubsection{Existence and Positivity of Solutions}

In this section, it is shown that the malaria model governed by the system of equations (1) to (6) is epidemiologically and mathematically well posed. Specifically, the feasible region is identified as $\Omega=\left\{\Omega_{\mathrm{h}} \times \Omega_{\mathrm{v}}\right\} \subset\left\{\mathbb{R}_{+}^{3} \times \mathbb{R}_{+}^{3}\right\}$ where $\Omega_{\mathrm{h}}=$ $\left\{\left(\mathrm{S}_{\mathrm{h}}, \mathrm{I}_{\mathrm{h}}, \mathrm{R}_{\mathrm{h}}\right) \in \mathbb{R}_{+}^{3}: \mathrm{N}_{\mathrm{h}} \leq\left(\Lambda_{\mathrm{h}} / \mu_{\mathrm{h}}\right)\right\} \quad$ and $\quad \Omega_{\mathrm{v}}=$ $\left\{\left(\mathrm{S}_{\mathrm{v}}, \quad \mathrm{I}_{\mathrm{vs}}, I_{v r}\right) \in \mathbb{R}_{+}^{3}: \mathrm{N}_{\mathrm{v}} \leq\left[\Lambda_{\mathrm{v}} / \mu_{v}\right]\right\}$.

Theorem 1 The solution $\left\{S_{h}, I_{h}, R_{h}, S_{v}, I_{v s} I_{v r}\right\}$ of the system of equations (1) to (6) is bounded and contained in the domain $\Omega$.

Proof: Let the solution of the system of equations (1) to (6) together with positive the initial conditions given in (9) are $\Omega=\left\{\mathrm{S}_{\mathrm{h}}, \mathrm{I}_{\mathrm{h}}, \mathrm{R}_{\mathrm{h}}, \mathrm{S}_{\mathrm{v}}, \quad \mathrm{I}_{\mathrm{vs}} I_{v r}\right\}$. Also, let $N_{h}(t)=$ $S_{h}(t)+I_{h}(t)+R_{h}(t)$ and $N_{v}(t)=S_{v}(t)+I_{v s}(t)+I_{v r}$. Now, in order to show that both the human and mosquito populations are bounded it is enough to show that the respective two total populations i.e., $N_{h}(t)$ and $N_{v}(t)$ are bounded.

Boundedness of $N_{h}(t)$ : Addition of human compartments of the system of equations (1) to (3) leads to $d N_{h} / d t=$ $\Lambda_{\mathrm{h}}-\mu_{\mathrm{h}} N_{h}(t)-\delta_{h} I_{h}(t)$. After dropping the negative term $-\delta_{h} I_{h}(t)$ appearing on the right-hand side the fore going equation can be expressed without loss of generality as an inequality as $d N_{h} / d t \leq \Lambda_{\mathrm{h}}-\mu_{\mathrm{h}} N_{h}(t)$ or equivalentl yas $d N_{h} / d t+\mu_{\mathrm{h}} N_{h}(t) \leq \Lambda_{\mathrm{h}}$. It is a first order linearordinary differential equation and has the generals olution $N_{h}(t) \leq$ $\left(\Lambda_{\mathrm{h}} / \mu_{\mathrm{h}}\right)+A \exp \left(-\mu_{\mathrm{h}} \mathrm{t}\right)$. Here, the integral constant $A$ takes theform, on applying the initial conditions as $A=$ $\left[N_{0 h}-\left(\Lambda_{\mathrm{h}} / \mu_{\mathrm{h}}\right)\right]$. Hence, the complete solution is given by $N_{h}(t) \leq\left(\Lambda_{\mathrm{h}} / \mu_{\mathrm{h}}\right)+\left[N_{0 h}-\left(\Lambda_{\mathrm{h}} / \mu_{\mathrm{h}}\right)\right] \exp \left(-\mu_{\mathrm{h}} \mathrm{t}\right)$. Now, clearly it can be observed that $N_{h}(t) \leq\left(\Lambda_{\mathrm{h}} / \mu_{\mathrm{h}}\right)$ as $t \rightarrow \infty$ and also, according to the initial conditions $N_{h}(t)=N_{h}$ at the initial timet $=0$. Hence, the total human population is bounded i.e. $N_{0 h} \leq N_{h}(t) \leq\left(\Lambda_{\mathrm{h}} / \mu_{\mathrm{h}}\right)$.

Boundedness of $N_{v}(t)$ : Addition of mosquito compartments of the system of equations (1) to (6) leads to $d N_{v} / d t=\Lambda_{v}-\mu_{v} N_{v}-\alpha\left(S_{v}+I_{v s}\right)-\delta_{v}\left(I_{v} s+I_{v r}\right)$. After dropping the negative term $-\alpha\left(S_{v}+I_{v s}\right)-$ $\delta_{v}\left(I_{v s}+I_{v r}\right)$ appearing on the right-hand side, the fore going equation can be expressed without loss of generality as an inequality as. $d N_{v} / d t \leq \Lambda_{v}-\mu_{v} N_{v}(t)$, or equivalently it is $d N_{v} / d t+\mu_{\mathrm{v}} N_{v}(t) \leq \Lambda_{v}$. It is a first order linear ordinary differential equation and has the general solution $N_{v}(t) \leq$ $\left[\Lambda_{\mathrm{v}} / \mu_{\mathrm{v}}\right]+B \exp \left[\left(-\mu_{\mathrm{v}}\right) \mathrm{t}\right]$. Here, the integral constant $B$ takes the form, on applying the initial condition, as $B=$ $\left[N_{0 v}-\left(\Lambda_{\mathrm{v}} / \mu_{\mathrm{v}}\right)\right]$. Hence, the complete solution is given by $N_{h}(t) \leq\left(\Lambda_{\mathrm{v}} / \mu_{\mathrm{v}}\right)+\left[N_{0 v}-\left(\Lambda_{\mathrm{v}} / \mu_{\mathrm{v}}\right)\right] \exp \left(-\mu_{\mathrm{v}} \mathrm{t}\right)$. Now, clearly it can be observed that $N_{v}(t) \leq\left[\Lambda_{\mathrm{v}} / \mu_{\mathrm{v}}\right]$ as $t \rightarrow \infty$ and also according to the initial condition $N_{0}(t)=N_{0 v}$ at the initial time $t=0$. Hence, the total mosquito population is bounded i.e. $N_{0 v} \leq N_{v}(t) \leq\left[\Lambda_{\mathrm{v}} / \mu_{\mathrm{v}}\right]$.

Thus, the solutions of the model variables representing human populations $\left\{S_{h}(t), I_{h}(t), R_{h}(t)\right\}$ are confined in the feasible region $\Omega_{\mathrm{h}}=\left\{\left(\mathrm{S}_{\mathrm{h}}, \mathrm{I}_{\mathrm{h}}, \quad \mathrm{R}_{\mathrm{h}}\right) \in \mathbb{R}_{+}^{3}: \mathrm{N}_{\mathrm{h}} \leq\left(\Lambda_{\mathrm{h}} / \mu_{\mathrm{h}}\right)\right\}$ Similarly, the solutions of the model variables representing mosquito populations $\left\{\left(S_{v}, I_{v s} I_{v r}\right)\right\}$ are confined in the feasible region $\Omega_{\mathrm{v}}=\left\{\left(\mathrm{S}_{\mathrm{v}}, \quad \mathrm{I}_{\mathrm{vs}} I_{v r}\right) \in \mathbb{R}_{+}^{3}: \mathrm{N}_{\mathrm{v}} \leq\left[\Lambda_{v} / \mu_{v}\right]\right\}$. This shows that the feasible region of the model equations (1) to (6) is bounded and is given by $\Omega=\left\{S_{h}(t), \quad I_{h}(t), \quad R_{h}(t), \quad S_{v}(t), \quad I_{v s}(t) \quad I_{v r}(t)\right\} \in \mathbb{R}_{+}^{6}$ or equivalently $\Omega=\left\{\Omega_{\mathrm{h}} \times \Omega_{\mathrm{v}}\right\} \subset\left\{\mathbb{R}_{+}^{3} \times \mathbb{R}_{+}^{3}\right\}$.

Positivity of the model equations is verified. The results are stated and proved in the form of a theorem as follows:

Theorem 2: The solutions $\left\{S_{h}(t), \quad I_{h}(t), \quad R_{h}(t), \quad S_{v}(t), \quad I_{v s}(t) \quad I_{v r}(t)\right\} \quad$ of the malaria model given in equations (1) to (6) together with the non-negative initial conditions given in (1)are all nonnegative for all $t>0$.

Proof:

Positivity of $S_{h}$ : Consider the equation for susceptible humans from the system of equations (1) to (6) i.e. $d S_{h} / d t=$ $\Lambda_{h}+\theta R_{h}-\left[\beta_{h}\left(1-\beta_{h}\right)\left(I_{v s}+I_{v s}\right) S_{h}\right] / N_{h}-\mu_{h} S_{h}$. After dropping the positive terms $\Lambda_{h}$ and $\theta R_{h}$ appearing on the right-hand side, the fore going equation can be expressed without loss of generality as an inequality $d S_{h} / d t \geq$ $-S_{h}\left[\beta_{h}\left(1-\beta_{h}\right)\left(I_{v s}+I_{v r}\right)\right] / N_{h}-\mu_{h} S_{h}$. Also $N_{h}$ satisfies the boundary condition

$N_{h} \leq\left(\Lambda_{h} / \mu_{h}\right)$ as showninTheorem1Thus, it can be equivalently expressed as:

$d S_{h} / d t \geq-S_{h}\left[\left[\left[\beta_{h}\left(1-\beta_{h}\right)\left(I_{v s}+I_{v r}\right)\right] \mu_{h} / \Lambda_{h}\right]+\mu_{h}\right]$. It is a first order linear ordinary differential equation and has the general solution:

$S_{h}(t) \geq \exp \left[S_{0 h}-\left[\left[\beta_{h}\left(1-\beta_{h}\right)\left(I_{0 v s}+I_{0 v r}\right) \mu_{h} / \Lambda_{h}\right]+\right.\right.$ $\left.\left.\mu_{h}\right] t\right] \geq 0$. Therefore $S_{h}(t) \geq 0$ for all $t>0$.

Positivity of $I_{h}$ : Consider the equation for infected humans from the system of equations (1) to (6) i.e. $d I_{h} / d t=$ $\left[\left[\beta_{h}\left(1-\beta_{h}\right)\left(I_{v s}+I_{v r}\right)\right] \mu_{h} / N_{h}\right]-\left(\gamma+\mu_{h}+\delta_{h}\right) I_{h}$. After dropping the positive term $\left[\beta_{h}\left(1-\beta_{h}\right)\left(I_{v s}+I_{v r}\right)\right] \mu_{h} / N_{h}$ appearing on the right-hand side the fore going equation it can be expressed without loss of generality as an inequality $d I_{h} / d t \geq-\left[\gamma+\mu_{h}+\delta_{h}\right] I_{h}$. It is a first order linear ordinary differential equation and has the general solution. $I_{h}(t)=\exp \left[I_{0 h}-\left(\gamma+\mu_{h}+\delta_{h}\right) t\right] \geq$ 0 . Therefore $I_{h}(t) \geq 0$ for all $t>0$.

Positivity of $R_{h}$ : Consider the equation for recovered humans from the system of equations (1) to (6) i.e. $d R_{h} / d t=\gamma I_{h}-\left(\mu_{h}+\theta\right) R_{h}$. After dropping the positive term $\gamma I_{h}$ appearing on the right-hand side the fore going equation can be expressed without loss of generality as an inequality $d R_{h} / d t \geq-\left(\mu_{h}+\theta\right) R_{h}$. It is a first order linear ordinary differential equation and has the general solution $R_{h}(t)=\exp \left[R_{0 h}-\left(\mu_{h}+\theta\right) t\right]>0$. Therefore $R_{h}(t) \geq 0$ for all $t>0$.

Positivity of $S_{v}$ : Consider the equation for susceptible vector from the system of equations (1) to (6) i.e. $d S_{v} / d t=$ 
$\Lambda_{v}-\left[\beta_{v} S_{v} I_{h}\right] / N_{v}-\left(\alpha+\mu_{v}\right) S_{v}$. After dropping the positive term $\Lambda_{v}$ appearing on the right-hand side, the fore going equation can be expressed without loss of generality as $d S_{v} / d t \geq-S_{v}\left[\left[\beta_{v} I_{h} / \mathrm{N}_{v}\right]+\left(\alpha+\mu_{v}\right]\right.$. Since $\mathrm{N}_{\mathrm{v}} \leq$ $\Lambda_{v} / \mu_{v}$ and hence, it can be equivalently expressed as $d S_{v} / d t \geq-S_{v}\left[\left(\mu_{v} \beta_{v} I_{h} / \Lambda_{v}\right)+\left(\alpha+\mu_{v}\right)\right]$. It is a first order linear ordinary differential equation and has the general solution $S_{v}(t)=\exp \left[S_{0 v}-\left[\left[\mu_{v} \beta_{v} I_{0 h} / \Lambda_{v}\right]+\left(\alpha+\mu_{v}\right)\right] t\right]>$ 0 . Therefore $S_{v}(t) \geq 0$ for all $t>0$.

Positivity of $I_{v s}$ : Consider the equation for insecticide sensitive malariavectors from the system of equations (1) to (6) i.e. $d I_{v s} / d t=\left[\rho(1-\varphi) \beta_{v} S_{v} I_{h} / N_{v}\right]-\left(\alpha+\mu_{v}+\right.$ $\left.\delta_{v}\right) I_{v s}$. After dropping the positive term $\left[\rho(1-\varphi) \beta_{v} S_{v} I_{h} / N_{v}\right]$ which is appearing on the righthand side, the fore going equation can be expressed without of loss of generality as $d I_{v s} / d t \geq-\left(\alpha+\mu_{v}+\delta_{v}\right) I_{v s}$. It is a first order linear ordinary differential equation and has solution $I_{v s}(t)=\exp \left[I_{0 v s}-\left(\alpha+\mu_{v}+\delta_{v}\right) t\right]>0$. Therefore, $I_{v s}(t) \geq 0$ for all $t>0$.

Positivity of $I_{v r}$ : Consider the equation for insecticideresistant malaria vectors from the system of equations (1) to (6) i.e. $d I_{v r} / d t=\left[[1-(1-\varphi) \rho] \beta_{v} S_{v} I_{h}\right] / N_{v}-\left(\mu_{v}+\delta_{v}\right) I_{v r}$. After dropping the positive term $\left\{[1-(1-\varphi) \rho] \beta_{v} S_{v} I_{h} / N_{v}\right\}$ which is appearing on the righthand side, the fore going equation can be expressed without loss of generality as $d I_{v s} / d t \geq-\left(\mu_{v}+\delta_{v}\right) I_{v r}$. It is a first order linearordinary differential equation and has the general solution $I_{v r}(t)=\exp \left[I_{0 v r}-\left(\mu_{v}+\delta_{v}\right) t\right]>0$. Therefore, $I_{v r}(t) \geq 0$ for all $t>0$.

\subsubsection{Existence of Equilibriumpoints}

In this section, the model is analyzed quantitatively by investigating the existence and stability of both Disease-free equilibrium $E_{0}$ and at endemic equilibrium $E^{*}$.

The disease-free equilibrium points of the model are its steady state solutions in the absence of infection or disease. Consider the disease free-equilibrium points denoted by $E_{0}=\left\{S_{h}^{0}, I_{h}^{0}, R_{h}^{0}, S_{v}^{0}, I_{v s}^{0}, I_{v r}^{0}\right\}$. All the components of $E_{0}$ are obtained by setting $R_{h}^{0}=0, I_{v s}^{0}=0, I_{v r}^{0}=0, I_{h}^{0}=$ 0 in the malaria model equations (1) to (6)and solving the resultant equations: $d S_{h} / d t=0$ gives $S_{h}^{0}=\Lambda_{h} / \mu_{h}$ and similarly $d S_{v} / d t=0$ gives $S_{v}^{0}=\Lambda_{v} / \mu_{v}$. Thus,

$$
E_{0}=\left\{\Lambda_{h} / \mu_{h}, \quad 0, \quad 0, \Lambda_{v} / \mu_{v}, \quad 0,0\right\}
$$

Let the endemic equilibrium point be denoted by $E^{*}=$ $\left\{S_{h}^{*}, I_{h}^{*}, R_{h}^{*}, S_{v}^{*}, I_{v s}^{*}, I_{v r}^{*}\right\}$. It is the non-trivial positive equilibrium of the malaria model equations (1) to (6). Eachcomponent of $E^{*}$ is obtained by setting the right hand sides of all equations (1) to (6) equal to zero i.e.

$$
\begin{gathered}
\Lambda_{h}+\theta R_{h}-\left[\beta_{h}\left(1-\beta_{h}\right)\left(I_{v s}+I_{v s}\right) S_{h}\right] / N_{h}-\mu_{h} S_{h}=0 \\
{\left[\beta_{h}\left(1-\beta_{h}\right)\left(I_{v s}+I_{v s}\right) S_{h}\right] / N_{h}-\left(\gamma+\mu_{h}+\delta_{h}\right) I_{h}=0} \\
\gamma I_{h}-\left(\mu_{h}+\theta\right) R_{h}=0 \\
\Lambda_{v}-\left[\beta_{v} S_{v} I_{h}\right] / N_{v}-\left(\alpha+\mu_{v}\right) S_{v}=0 \\
{\left[\left(\rho(1-\varphi) \beta_{v}\right) S_{v} I_{h}\right] / N_{v}-\left(\alpha+\mu_{v}+\delta_{v}\right) I_{v s}=0}
\end{gathered}
$$

$$
\left[(1-(1-\varphi) \rho) \beta_{v} S_{v} I_{h}\right] / N_{v}-\left(\mu_{v}+\delta_{v}\right) I_{v r}=0
$$

Up on computing the resultant equations as listed above, thecomponents of $E^{*}$ are obtained as follows:

$$
\begin{gathered}
S_{h}^{*}=\frac{\Lambda_{h}\left(\mu_{h}+\theta\right)+\left[\theta \gamma-\left(\mu_{h}+\theta\right)\left(\gamma+\mu_{h}+\delta_{h}\right)\right] I_{h}^{*}}{\mu_{h}\left(\mu_{h}+\theta\right)} \\
I_{h}^{*}=\frac{\Lambda_{v}\left(\mu_{h}+\theta\right)\left[\left[\Lambda_{h} \Lambda_{v}\left(\gamma+\mu_{h}+\delta_{h}\right) R_{e}^{2}\right]-\left(\alpha+\mu_{v}\right)\right]}{R_{e}^{2} \Lambda_{v}^{2}\left[\left(\mu_{h}+\theta\right)\left(\gamma+\mu_{h}+\delta_{h}\right)-\theta \gamma\right]+\left(\mu_{h} \mu_{v} \beta_{v}\right)\left(\mu_{h}+\theta\right)} \\
R_{h}^{*}=\frac{\gamma I_{h}^{*}}{\left(\mu_{h}+\theta\right)} \\
S_{v}^{*}=\frac{\Lambda_{v}}{\beta_{v} I_{h}^{*}+\left(\alpha+\mu_{v}\right)} \\
I_{v S}^{*}=\frac{\Lambda_{v} \beta_{v}[(1-\varphi) \rho]}{\left(\alpha+\mu_{v}+\delta_{v}\right)\left[\beta_{v} I_{h}^{*}+\left(\alpha+\mu_{v}\right)\right]} \\
I_{v r}^{*}=\frac{\Lambda_{v} \beta_{v}[(1-(1-\varphi) \rho]}{\left(\mu_{v}+\delta_{v}\right)\left[\beta_{v} I_{h}^{*}+\left(\alpha+\mu_{v}\right)\right]}
\end{gathered}
$$

\subsubsection{Reproduction Number}

The basic reproduction number denoted by $R_{0}$ is the average number of secondary infectious infected by an infective individual during his or her whole course of disease in case that all of the population are susceptible [25]. This helps to check whether an infection will spread through the population or die out from the population.

\subsubsection{The Effective Reproduction Number}

The effective reproduction number $R_{e}$ is a key parameter that determines the behavior of the model in the presence of indoor residual spray IRS. The term 'effective reproduction number' is used to distinguish it from the basic reproduction number $R_{0}$. The latter is used when there is no indoor residual spray IRS.

In order to analyze the stability of system $2 \mathrm{a}$-fthe threshold condition for the establishment of the disease is required tobe obtained.

Here the effective reproduction number is computed using the next generation operator method that is developed by van den Driessche and Watmough [26]. A reproduction number obtained this way determines the local stability of the disease-free equilibrium point with local asymptotic stability for $R_{e}<1$ and instability for $R_{e}>1$. Now let the system be rearranged by beginning with the infected classes as follows: Let $X=$ $\left(\mathrm{I}_{\mathrm{h}}, \mathrm{I}_{\mathrm{vs}}, \mathrm{I}_{\mathrm{vr}}, \mathrm{S}_{\mathrm{h}}, R_{h}, S_{v}\right)^{T}$. Then the new infections be distinguished from all other class transitions in the population.

The infected classes are $\mathrm{I}_{\mathrm{h}}, \mathrm{I}_{\mathrm{vs}}$ and $\mathrm{I}_{\mathrm{vr}}$ among all the classes of both human host and mosquito vector. The vector of rates of the appearance of new infections in each compartment is denoted by $F$. Further, $V=V^{+}+V^{-}$where $V^{+}$is the vector rate of transfer into the particular compartment and $V^{-}$is the vector rateoftransfer out of the particular compartment. In the model equations it is clear that there are three compartments for the infected. Thus, 


$$
F\left(X_{i}\right)=\left[\begin{array}{c}
\beta_{h}\left(1-\beta_{h}\right) S_{h}\left(I_{v r}+I_{v s}\right) / N_{h} \\
\rho \beta_{v} S_{v} I_{h} / N_{v} \\
{[1-(1-\varphi) \rho] \beta_{v} S_{v} I_{h} / N_{v}} \\
0 \\
0 \\
0
\end{array}\right] \text { and } V\left(X_{i}\right)=
$$

Now, the matrices $F$ and $V$ at the disease-free equilibrium point $E_{0}$ are defined as $F=\frac{\partial F\left(X_{i}\right)}{\partial X_{i}}\left(E_{0}\right)$ and $V=\frac{\partial V\left(X_{i}\right)}{\partial X_{i}}\left(E_{0}\right)$. After computation they are obtained as

$$
F=\left[\begin{array}{ccc}
0 & \beta_{h}\left(1-\beta_{h}\right) & \beta_{h}\left(1-\beta_{h}\right) \\
{[(1-\varphi) \rho] \beta_{v}} & 0 & 0 \\
{[1-(1-\varphi) \rho] \beta_{v}} & 0 & 0
\end{array}\right]
$$

and

$$
V=\left[\begin{array}{ccc}
\left(\gamma+\mu_{\mathrm{h}}+\delta_{\mathrm{h}}\right) & 0 & 0 \\
0 & \left(\mu_{v}+\delta_{v}+\alpha\right) & 0 \\
0 & 0 & \left(\mu_{v}+\delta_{v}\right)
\end{array}\right]
$$

Note that $\mathrm{F}$ is nonnegative, $\mathrm{V}$ is a nonsingular matrix whose inverse $\mathrm{V}^{-1}$, is nonnegative, and the next generation matrix and $\mathrm{FV}^{-1}[25,26]$ is nonnegative. The dominant eigenvalue corresponding to the spectral radius $\left(\mathrm{FV}^{-1}\right)$ of the matrix

$$
F V^{-1}=\left[\begin{array}{ccc}
0 & \frac{\beta_{h}\left(1-\beta_{h}\right)}{\left(\mu_{v}+\delta_{v}+\alpha\right)} & \frac{\beta_{h}\left(1-\beta_{h}\right)}{\left(\mu_{v}+\delta_{v}\right)} \\
\frac{[(1-\varphi) \rho] \beta_{v}}{\left(\gamma+\mu_{h}+\delta_{h}\right)} & 0 & 0 \\
\frac{[1-(1-\varphi) \rho] \beta_{v}}{\left(\gamma+\mu_{h}+\delta_{h}\right)} & 0 & 0
\end{array}\right]
$$

$F V^{-1}$ is given by computing the corresponding characteristic equationdet $\left(F V^{-1}-\lambda I_{6}\right)=0$ which gives the effective reproduction numberdenoted andgivenby $R_{e}$, where

$$
R_{e}=\sqrt{\frac{\beta_{h}\left(1-\beta_{h}\right)\left[\left(\mu_{v}+\delta_{v}\right)((1-\varphi) \rho)+\left(\mu_{v}+\delta_{v}+\alpha\right)(1-(1-\varphi) \rho)\right]}{\left(\gamma+\mu_{h}+\delta_{h}\right)\left(\mu_{v}+\delta_{v}+\alpha\right)\left(\mu_{v}+\delta_{v}\right)}}
$$

The effective reproduction number, $R_{e}$ is defined as the number of secondary infections derived from a single primary infection in a population of susceptible [26, 27]. Its biological meaning is readily interpreted from sum of the terms denoted by $R_{e s}$ and $R_{e r}$ in which

$$
R_{v s}=\sqrt{\frac{\beta_{h}\left(1-\beta_{h}\right) \beta_{v}((1-\varphi) \rho)}{\left(\gamma+\mu_{h}+\delta_{h}\right)\left(\mu_{v}+\delta_{v}+\alpha\right)}} \text { and } R_{v r}=\sqrt{\frac{\beta_{h}\left(1-\beta_{h}\right) \beta_{v}[(1-(1-\varphi) \rho)]}{\left(\gamma+\mu_{h}+\delta_{h}\right)\left(\mu_{v}+\delta_{v}\right)}}
$$

If there is no any control strategy i.e., $\alpha=0$, then the effective reproduction number $R_{e}$ for the model equations $2 \mathrm{a}$-freduces to the basic reproduction number denoted and is given by

$$
R_{o}=\sqrt{\frac{\beta_{h}\left(1-\beta_{h}\right) \beta_{v}[((1-\varphi) \rho)+(1-(1-\varphi) \rho)]}{\left(\gamma+\mu_{h}+\delta_{h}\right)\left(\mu_{v}+\delta_{v}\right)}}
$$

\subsubsection{Local Stability of the Disease-Free Equilibrium Point}

Here, the stability analysis of the disease-free Equilibrium point $E_{0}=\left\{\begin{array}{llllllll}S_{h}^{0}, & I_{h}^{0}, & R_{h}^{0}, & S_{v}^{0} & I_{v s}^{0} & I_{v r}^{0}\end{array}\right\}$ of model equations (1) to (6) computing its Jacobian matrix. The Jacobian matrix is computed by differentiating the left-hand side function of each equation in the system with respect to the state variables $S_{h}, I_{h}, R_{h}, \quad S_{v} I_{v s} I_{v r}$. That is, the followingsystem of modelequations will be considered to construct Jacobian matrix and to conduct further analysis.

The stability analysis and the results are stated and proved in Theorem 3.

$$
\begin{array}{r}
d S_{h} / d t=\Lambda_{h}+\theta R_{h}-\left[\beta_{h}\left(1-\beta_{h}\right)\left(I_{v s}+I_{v s}\right) S_{h}\right] / N_{h}-\mu_{h} S_{h} \\
d I_{h} / d t=\left[\beta_{h}\left(1-\beta_{h}\right)\left(I_{v s}+I_{v s}\right) S_{h}\right] / N_{h}-\left(\gamma+\mu_{h}+\delta_{h}\right) I_{h} \\
d R_{h} / d t=\gamma I_{h}-\left(\mu_{h}+\theta\right) R_{h} \\
d S_{v} / d t=\Lambda_{v}-\left[\beta_{v} S_{v} I_{h}\right] / N_{v}-\left(\alpha+\mu_{v}\right) S_{v} \\
d I_{v s} / d t=\left[[\rho(1-\varphi)] \beta_{v} S_{v} I_{h}\right] / N_{v}-\left(\alpha+\mu_{v}+\delta_{v}\right) I_{v s} \\
d I_{v r} / d t=\left[[1-(1-\varphi) \rho] \beta_{v} S_{v} I_{h}\right] / N_{v}-\left(\mu_{v}+\delta_{v}\right) I_{v r}
\end{array}
$$

Theorem 3: The Disease-free Equilibrium point $E_{0}$ is locally asymptotically stableif $R_{e}<1$ but unstable if $R_{e}>1$.

Proof:

The Jacobian matrix of the system of equations (1) to (6) evaluated at the disease-free equilibrium point $E_{0}$ is given by:

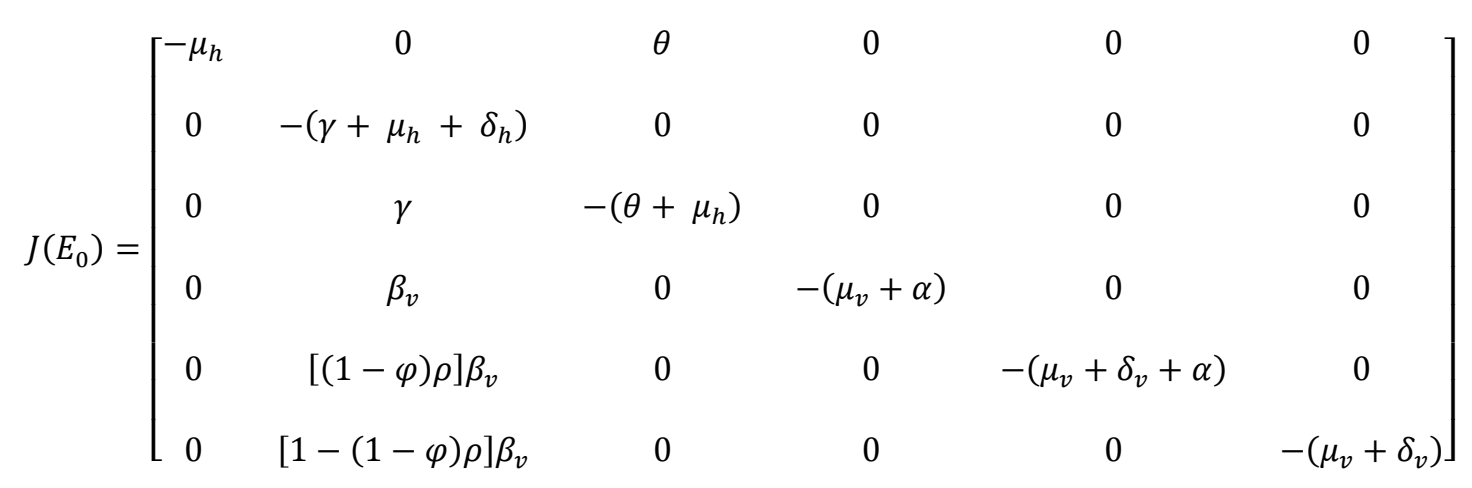


In order to prove the statement, it is required to show that all the eigenvalues of $J\left(E_{0}\right)$ are negative. Since the

first and fourth, fifth, and sixth columns contain only diagonal terms they givefour negative eigenvalues $\lambda_{1}=$ $-\mu_{h}, \lambda_{2}=-\left(\mu_{v}+\alpha\right) \quad, \quad \lambda_{3}=-\left(\mu_{v}+\delta_{v}+\alpha\right), \lambda_{4}=$ $-\left(\mu_{v}+\delta_{v}\right)$. The other two eigenvalues can be computed from the sub-matrix $J_{1}\left(E_{0}\right)$ formed by excluding the first and the third rows and columns of $J\left(E_{0}\right)$. Hence $J_{1}\left(E_{0}\right)$ is given by

$$
\begin{gathered}
J_{1}\left(E_{0}\right)=\left[\begin{array}{cc}
-\left(\gamma+\mu_{h}+\delta_{h}\right) & 0 \\
\gamma & -\left(\theta+\mu_{h}\right)
\end{array}\right] \begin{array}{c}
\lambda^{2}+\left(2 \mu_{h}+\delta_{h}+\gamma+\theta\right) \lambda+\left(\mu_{h}+\delta_{h}+\gamma\right)\left(\theta+\mu_{h}\right)=0 \\
\text { Upon solving the quadratic equation, the } 5^{\mathrm{rd}} \text { and } 6^{\text {th }} \\
\text { eigenvalues } \lambda_{5} \text { and } \lambda_{6} \text { are obtained as }
\end{array} \\
\lambda_{5}=-\left\{\left(2 \mu_{h}+\delta_{h}+\gamma+\theta\right)+\sqrt{\left[\left(2 \mu_{h}+\delta_{h}+\gamma+\theta\right)^{2}-4\left(\mu_{h}+\delta_{h}+\gamma\right)\left(\theta+\mu_{h}\right)\right]}\right\} / 2 \\
\lambda_{6}=-\left\{\left(2 \mu_{h}+\delta_{h}+\gamma+\theta\right)-\sqrt{\left[\left(2 \mu_{h}+\delta_{h}+\gamma+\theta\right)^{2}-4\left(\mu_{h}+\delta_{h}+\gamma\right)\left(\theta+\mu_{h}\right)\right]}\right\} / 2
\end{gathered}
$$

0 takes the form as:

$$
\left|\begin{array}{cc}
-\left(\mu_{h}+\delta_{h}+\gamma\right)-\lambda & 0 \\
\gamma & -\left(\theta+\mu_{h}\right)-\lambda
\end{array}\right|=0
$$

Also, the characteristic equation can be expressed in a quadratic form as
Here, it can be observed that the eigenvalue $\lambda_{5}$ is absolutely a negative. However, the eigenvalue $\lambda_{6}$ is a negative if the condition

$\sqrt{\left[\left(2 \mu_{h}+\delta_{h}+\gamma+\theta\right)^{2}-4\left(\mu_{h}+\delta_{h}+\gamma\right)\left(\theta+\mu_{h}\right)\right]} / 2<0$ is valid.

Thus, all the eigenvalues of the Jacobian matrix at the disease-free equilibrium $J\left(E_{0}\right)$ arenegative provided that $\left[\left(2 \mu_{h}+\delta_{h}+\gamma+\theta\right)^{2}-4\left(\mu_{h}+\delta_{h}+\gamma\right)\left(\theta+\mu_{h}\right)\right]<0 \quad$ if and only if $\frac{T^{2}}{R_{e}^{2}}\left(R_{e}^{2}-\frac{M K}{N T^{2}}\right)<0$ for which

$M K>N T^{2}$ or equivalently $R_{e}<1$ is valid, Where $K=$ $\left(\theta+\mu_{h}\right), N=\left(\mu_{v}+\delta_{v}+\alpha\right)\left(\mu_{v}+\delta_{v}\right)$

$$
\begin{gathered}
R_{e}=\sqrt{\frac{\beta_{h}\left(1-\beta_{h}\right) \beta_{v}\left[\left(\mu_{v}+\delta_{v}\right)((1-\varphi) \rho)+\left(\mu_{v}+\delta_{v}+\alpha\right)(1-(1-\varphi) \rho)\right]}{\left(\gamma+\mu_{h}+\delta_{h}\right)\left(\mu_{v}+\delta_{v}+\alpha\right)\left(\mu_{v}+\delta_{v}\right)}} T=\left(2 \mu_{h}+\delta_{h}+\gamma+\theta\right) \\
M=4 \beta_{h}\left(1-\beta_{h}\right)\left[\left(\mu_{v}+\delta_{v}\right)((1-\varphi) \rho)+\left(\mu_{v}+\delta_{v}+\alpha\right)(1-(1-\varphi) \rho)\right] \\
\left(\gamma+\mu_{h}+\delta_{h}\right)=\frac{\beta_{h}\left(1-\beta_{h}\right) \beta_{v}\left[\left(\mu_{v}+\delta_{v}\right)((1-\varphi) \rho)+\left(\mu_{v}+\delta_{v}+\alpha\right)(1-(1-\varphi) \rho)\right]}{R_{e}^{2}\left(\mu_{v}+\delta_{v}+\alpha\right)\left(\mu_{v}+\delta_{v}\right)}
\end{gathered}
$$

Therefore, the disease-free equilibrium point is locally asymptotically stable if $R_{e}<1$ and unstable if $R_{e}>1$.

Theorem 4. If $R_{e}<1$, then the disease free equilibrium point $E_{0}$ is globally asymptotically stable and unstable. if $R_{e}>1$.

Proof: Consider the following Lyapunov function to show the global stability of $E_{0}$.

$$
\begin{aligned}
& L\left[I_{h},\left(I_{v s}+I_{v r}\right)\right]=\left[\left(\mu_{v}+\delta_{v}\right) I_{h} / \beta_{h}\left(1-\beta_{h}\right)\right]-\left(I_{v s}+I_{v r}\right) \\
& d L / d t=-\left[\left(\mu_{v}+\delta_{v}\right) I_{h} / \beta_{h}\left(1-\beta_{h}\right)\right]\left(\gamma+\mu_{h}+\delta_{h}\right) I_{h}- \\
& \beta_{V} I_{h}+2\left(\mu_{v}+\delta_{v}\right)\left(I_{v s}+I_{v r}\right)+\alpha I_{v s} \\
& d L / d t=-\left[\left[\left(\mu_{v}+\delta_{v}\right) I_{h} / \beta_{h}\left(1-\beta_{h}\right)\right]\left(\gamma+\mu_{h}+\delta_{h}\right)\right. \\
& \left.-\beta_{V}\right] I_{h}+2\left(\mu_{v}+\delta_{v}\right)\left(I_{v s}+I_{v r}\right)+\alpha I_{v s} \\
& d L / d t \leq-\left[\left[\left(\mu_{v}+\delta_{v}\right) I_{h} / \beta_{h}\left(1-\beta_{h}\right)\right]\left(\gamma+\mu_{h}+\delta_{h}\right)-\right.
\end{aligned}
$$

$\left.\beta_{V}\right] I_{h} \leq 0$. Thus, $d L / d t \leq 0$. This is equivalent to $R_{e}<$ 1 from LaSalle's invariant principle [28]. Therefore, the disease-free equilibrium $\mathrm{E}_{0}$ is globally asymptotically stable in $\Omega$ if $R_{e}<1$.

\subsubsection{Local Stability of the Endemic Equilibrium Solution}

A disease is endemic in a given population if it continues to persistinthat population. The stability of endemic equilibrium of the model is studied in the following theorem.

Theorem5: The endemic equilibrium solution $E^{*}$ of the model equations (1) to (6) is locally asymptotically stable if $R_{e}>1$ and unstable if $R_{e}<1$.

Proof:

let $E^{*}=\left\{S_{h}^{*}, \quad I_{h}^{*}, \quad R_{h}^{*}, \quad S_{v}^{*}, \quad I_{v s}^{*}, \quad I_{v r}^{*}\right\}$ and $\operatorname{det}\left(J\left(E^{*}\right)-\right.$ $\left.\lambda I_{6}\right)=\left|\begin{array}{ll}J_{0} & J_{1} \\ J_{2} & J_{3}\end{array}\right|=0$ where

$$
J_{0}=\left|\begin{array}{ccc}
-\frac{\beta_{h}\left(1-\beta_{h}\right) \mu_{\mathrm{h}}\left(\mathrm{I}_{\mathrm{vs}}^{*}+\mathrm{I}_{\mathrm{vr}}^{*}\right)}{\Lambda_{\mathrm{h}}}-\mu_{\mathrm{h}}-\lambda & 0 & \theta \\
\frac{\beta_{h}\left(1-\beta_{h}\right) \mu_{\mathrm{h}}\left(I_{v s}^{*}+I_{v r}^{*}\right)}{\Lambda_{h}} & -\left(\gamma+\mu_{\mathrm{h}}+\delta_{h}\right)-\lambda & 0 \\
0 & \gamma & -\left(\theta+\mu_{\mathrm{h}}\right)-\lambda
\end{array}\right|=0
$$




$$
\begin{gathered}
J_{1}=\left|\begin{array}{ccc}
0 & -\frac{\beta_{h}\left(1-\beta_{h}\right) \mu_{h} S_{h}^{*} I_{v r}^{*}}{\Lambda_{h}} & -\frac{\beta_{h}\left(1-\beta_{h}\right) \mu_{h} S_{h}^{*} I_{v s}^{*}}{\Lambda_{h}} \\
0 & \frac{\beta_{h}\left(1-\beta_{h}\right) \mu_{h} S_{h}^{*} I_{v r}^{*}}{\Lambda_{h}} & \frac{\beta_{h}\left(1-\beta_{h}\right) \mu_{h} S_{h}^{*} I_{v s}^{*}}{\Lambda_{h}} \\
0 & 0 & 0
\end{array}\right|=0 \\
J_{2}=\left|\begin{array}{ccc}
0 & -\frac{\mu_{v} \beta_{v} S_{v}^{*}}{\Lambda_{v}} & 0 \\
0 & \frac{[(1-\varphi) \rho] \mu_{v} \beta_{v} S_{v}^{*}}{\Lambda_{v}} & 0 \\
0 & \frac{[1-(1-\varphi) \rho] \mu_{v} \beta_{v} S_{v}^{*}}{\Lambda_{v}} & 0
\end{array}\right|=0 \\
J_{3}=\left|\begin{array}{ccc}
\frac{\left[\beta_{v} I_{h}^{*}+\left(\mu_{v}+\alpha\right) \mu_{v}\right]}{\Lambda_{v}}-\lambda & 0 \\
\frac{[(1-\varphi) \rho] \mu_{v} \beta_{v} I_{h}^{*}}{\Lambda_{v}} & -\left(\mu_{v}+\delta_{v}+\alpha\right)-\lambda & 0 \\
\frac{[1-(1-\varphi) \rho] \mu_{v} \beta_{v} I_{h}^{*}}{\Lambda_{v}} & 0 & -\left(\mu_{v}+\delta_{v}\right)-\lambda
\end{array}\right|=0
\end{gathered}
$$

Here,

$\lambda_{1}=-\frac{\beta_{h}\left(1-\beta_{h}\right) \mu_{h}\left(I_{v S}^{*}+I_{v r}^{*}\right)}{\Lambda_{h}}-\mu_{h}=-\frac{\left(\gamma+\mu_{h}+\delta_{h}\right) \Lambda_{v} I_{h}^{*} R_{e}^{2}}{\beta_{v} I_{h}^{*}+\left(\mu_{v}+\alpha\right)}-\mu_{h}$

$\lambda_{2}=-\left(\gamma+\mu_{h}+\delta_{h}\right), \lambda_{3}=-\left(\theta+\mu_{h}\right)$ and $\lambda_{4}, \lambda_{5}$, and

$\lambda_{6}$ Can be estimatedorcomputed from the characteristicequation; that is,

$$
\lambda^{3}+d_{1} \lambda^{2}+d_{2} \lambda+d_{3}
$$

By Using the Routh-Hurwitz criterion, it can be seen that all the eigenvalues of the characteristic equation (20) have negative real part if and only if $d_{1}>0, d_{3}>0$ and $d_{1} d_{2}>$ $d_{3}[29]$.

Where $d_{1}=\left(\frac{\left(\gamma+\mu_{h}+\delta_{h}\right) \Lambda_{v} I_{h}^{*} R_{e}^{2}}{\beta_{v} I_{h}^{*}+\left(\mu_{v}+\alpha\right)}+\mu_{h}\right)+\left(\gamma+\mu_{h}+\delta_{h}\right)+$ $\left(\theta+\mu_{h}\right)$

$$
\begin{gathered}
d_{2}=\left(\frac{\left(\gamma+\mu_{h}+\delta_{h}\right) \Lambda_{v} I_{h}^{*} R_{e}^{2}}{\beta_{v} I_{h}^{*}+\left(\mu_{v}+\alpha\right)}+\mu_{h}\right)\left(2 \mu_{h}+\gamma+\delta_{h}+\theta\right) \\
+\left(\theta+\mu_{h}\right)\left(\gamma+\mu_{h}+\delta_{h}\right) \\
d_{3}=\left(\frac{\left(\gamma+\mu_{h}+\delta_{h}\right) \Lambda_{v} I_{h}^{*} R_{e}^{2}}{\beta_{v} I_{h}^{*}+\left(\mu_{v}+\alpha\right)}+\mu_{h}\right)\left(\theta+\mu_{h}\right)\left(\gamma+\mu_{h}+\delta_{h}\right) \\
+\frac{\left(\gamma+\mu_{h}+\delta_{h}\right) \Lambda_{v} I_{h}^{*} R_{e}^{2}}{\beta_{v} I_{h}^{*}+\left(\mu_{v}+\alpha\right)} \theta \gamma
\end{gathered}
$$

Clearly $d_{1}$ and $d_{3}$ are positive because both of them are a sum of positive variables but $d_{1} d_{2}>d_{3}$ if $\frac{\left(\gamma+\mu_{h}+\delta_{h}\right) \Lambda_{v} I_{h}^{*} R_{e}^{2}}{\beta_{v} I_{h}^{*}+\left(\mu_{v}+\alpha\right)} \theta \gamma<0$ equivalently $R_{e}-1$ must be positive which leads to $R_{e}>1$. Thus, the endemic equilibrium will be locally asymptotically stable if and only if $R_{e}>1$ and unstable if $R_{e}<1$.

\section{Simulations and Discussions}

In the present study an SIR-SImodel has been formulated and mathematically analyzed. The main objective of this study is to understand qualitatively the factors that has more impact on the efficacy of the incorporated control strategy for the transmission and spread of endemic malaria disease and its effectiveness on insecticide resistant and sensitive malaria vector strains. Here, simulation study is conducted in support of mathematical analysis. Numerical simulation of model system equations (1) to (6) is carried out using DE Discover Solver. The initial population sizes and a set of parameter values are chosen based on similar studies of [30, 31, 32]. Graphical representations showing the human and mosquito populations with and without control measure (IRS)are provided in Figures (2) - (3). And variations in reproduction numbers with respect to contact rate between the infected humans and the infected mosquitoes are provided in Figures (4) -(6). Since values of someparametersare not available in the real world, data from literature is used for some parameters and for others estimated values are assigned. Table 3 and 4show the values assigned tostate variables and parametersrespectively and these values have been used in conducting simulations study.

Table 3. Estimated values of state variables.

\begin{tabular}{lll}
\hline State variable & Initial value & Source \\
\hline $\mathrm{S}_{h}$ & 300 & {$[30,31,32]$} \\
$\mathrm{I}_{h}$ & 1 & {$[30,31,32]$} \\
$\mathrm{R}_{h}$ & 0 & {$[30,31,32]$} \\
$S_{v}$ & 250 & {$[30,31,32]$} \\
$\mathrm{I}_{v s}$ & 4 & Estimated \\
$\mathrm{I}_{v r}$ & 2 & Estimated \\
\hline
\end{tabular}


Table 4. Estimated values of parameters.

\begin{tabular}{lll}
\hline Parameter & Value & Source \\
\hline$\Lambda_{h}$ & 0.0280 & {$[30]$} \\
$\mu_{h}$ & 0.0000391 & {$[30]$} \\
$\delta_{h}$ & 0.00040 & {$[30]$} \\
$\beta_{h}$ & 0.4500 & Estimated \\
$\Lambda_{v}$ & 50.000 & Estimated \\
$\mu_{\mathrm{v}}$ & 0.04000 & {$[30]$} \\
$\delta_{\mathrm{v}}$ & 0.1000 & {$[30]$} \\
$\beta_{v}$ & 0.2150 & Estimated \\
$\theta$ & 0.0140 & Estimated \\
$\gamma$ & 0.2050 & {$[30]$} \\
$\alpha$ & 0.5000 & Estimated \\
$\rho$ & 0.2000 & Estimated \\
\hline
\end{tabular}

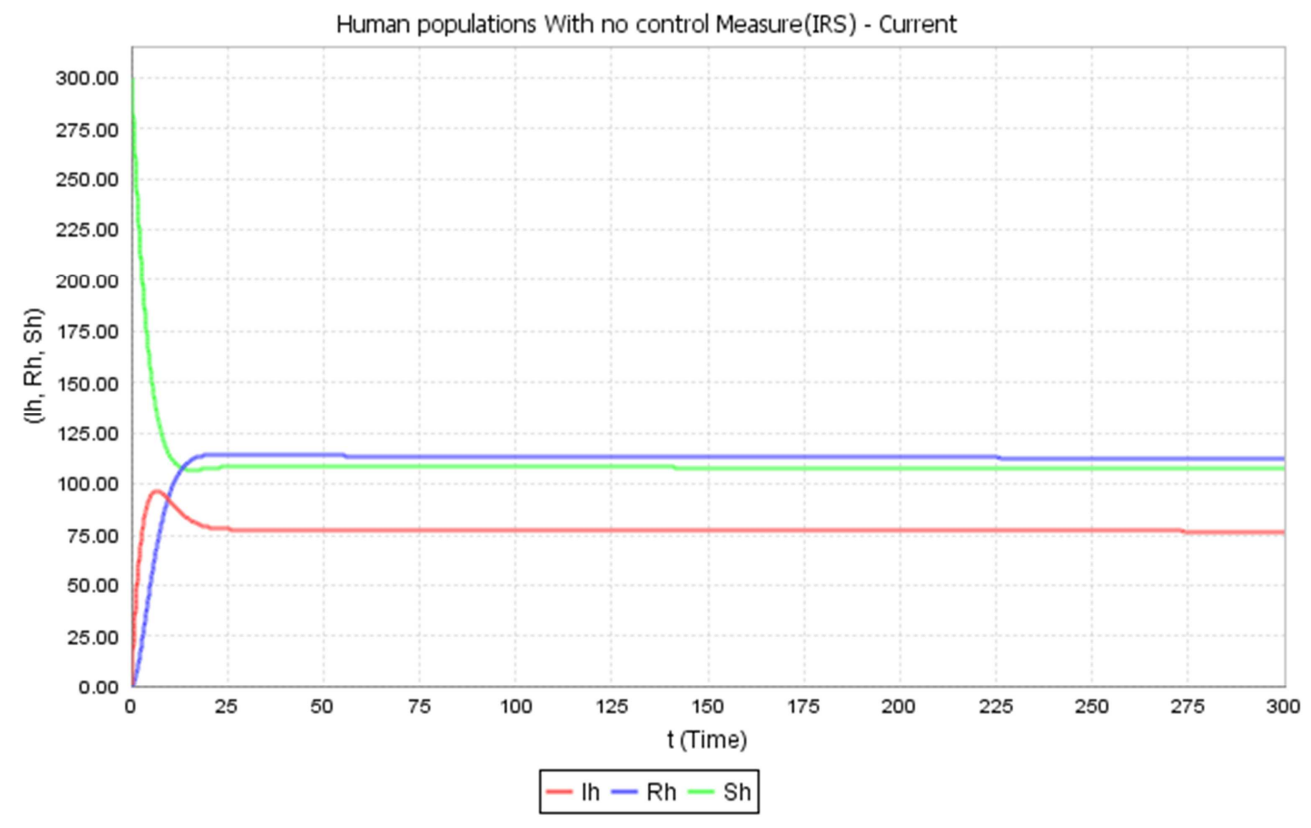

(a)

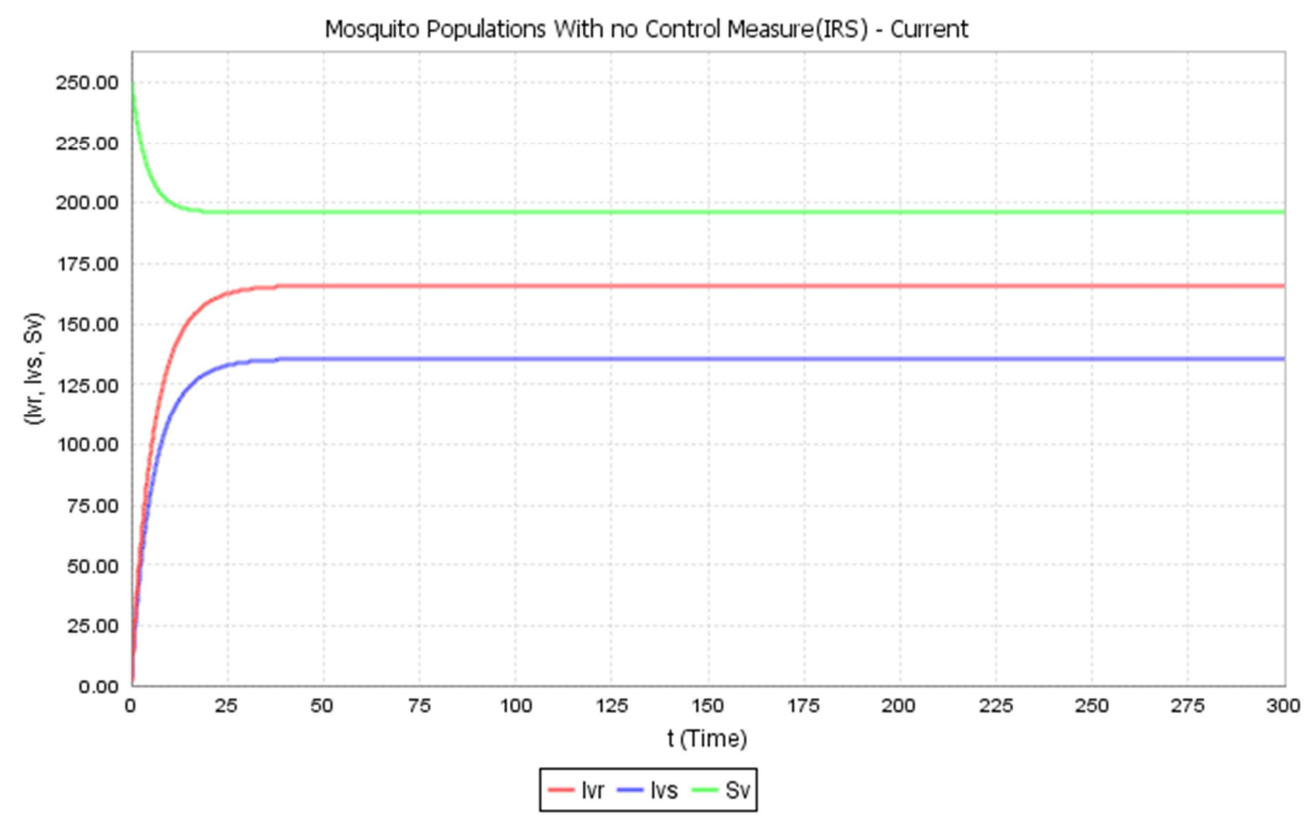

(b)

Figure 2. Human and Mosquito populations with no control measure (IRS). 
Figure 2 presents typical solution plots for the human populations and the mosquito populations. As the individuals leave one class for another, the curves change accordingly. Once the curves level off, we stop the simulation as the populations reached equilibrium. With no intervention strategy that is, when IRS $=0$, the final infected human populations is non-zero; however, infected human populations smaller than the susceptible and recovered classes of human populations. Since the populations are nonzero, malaria has not been eradicated at the equilibrium state.
For the mosquito classes, notice that the equilibrium state contains nearly almost an equal number of infected insecticide (IRS) resistant mosquito strains as susceptible mosquitoes while the infected insecticide sensitive mosquito strains are less in number than the insecticide(IRS) resistant mosquito strains. It is important to note that, here, the built in DE Discover solver will find a stable solution, which may have a dependence on initial conditions, if the system has more than one steady state.

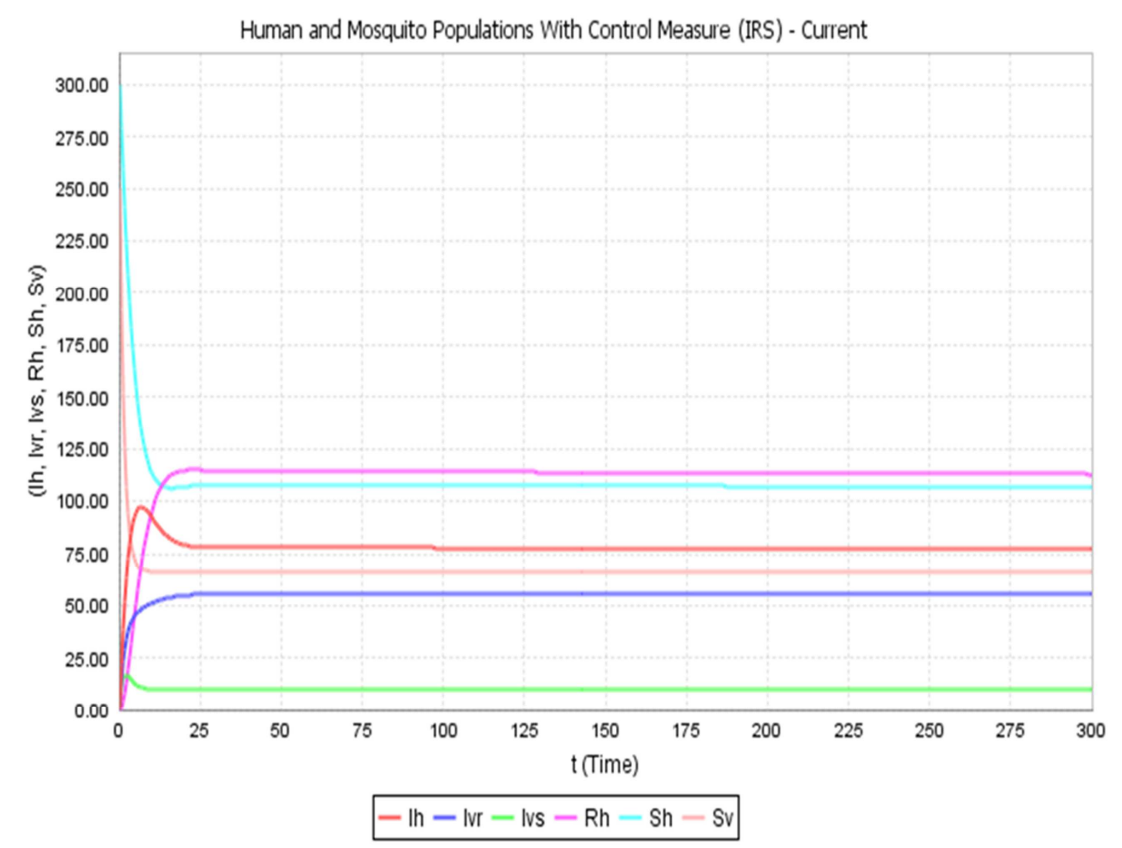

(a)

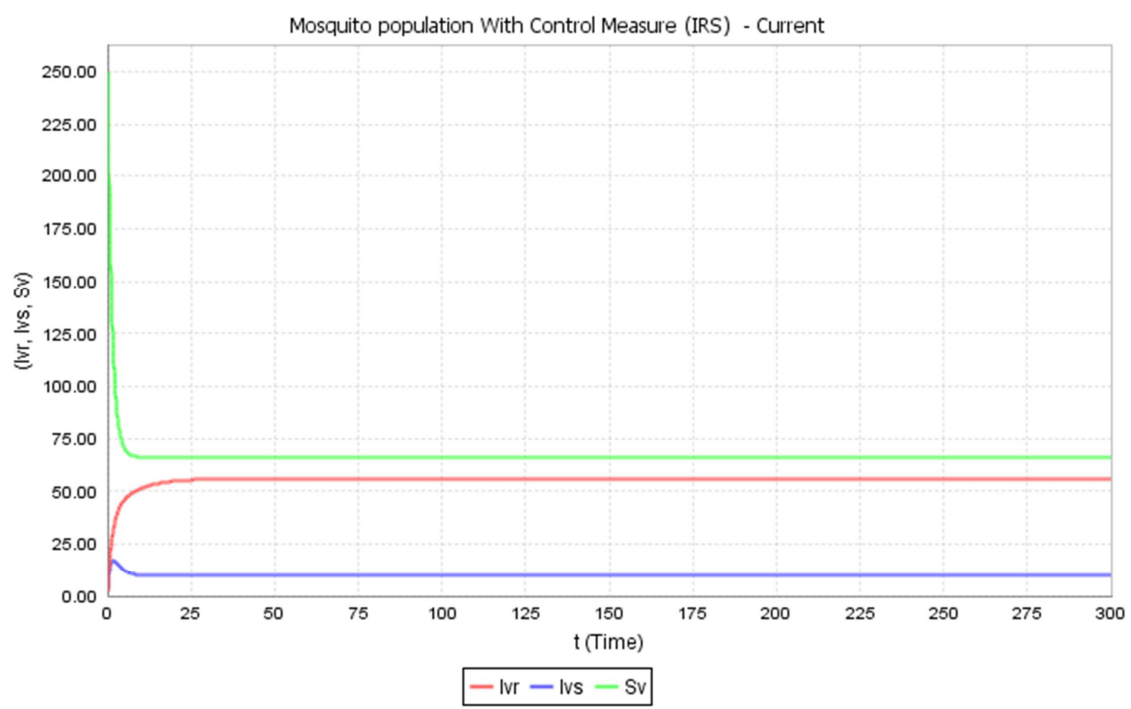

(b)

Figure 3. Human and Mosquito, and mosquito populations with control measure (IRS).

Figure 3 showthat the human and mosquito populations (top) and the mosquito populations separately (bottom) as a function of time. Thesefiguresare created using initial conditions from the Table 3, using the parameter values in Table 4. When IRS $=0.5$. The equations were solved in DE Discover solvers. Unlike Figure 2, In each mosquito 
populations classes therehas been seen a reduction of their numbers relatively. This is due to the fact that removal of mosquitoes from the different classes associated with IRS. Since the human populations are non-zero, malaria has not been eradicated at the equilibrium state. This phenomenon is due to the fact that the mosquito population becomes so small that the birth rate of humans greater than the death rate.

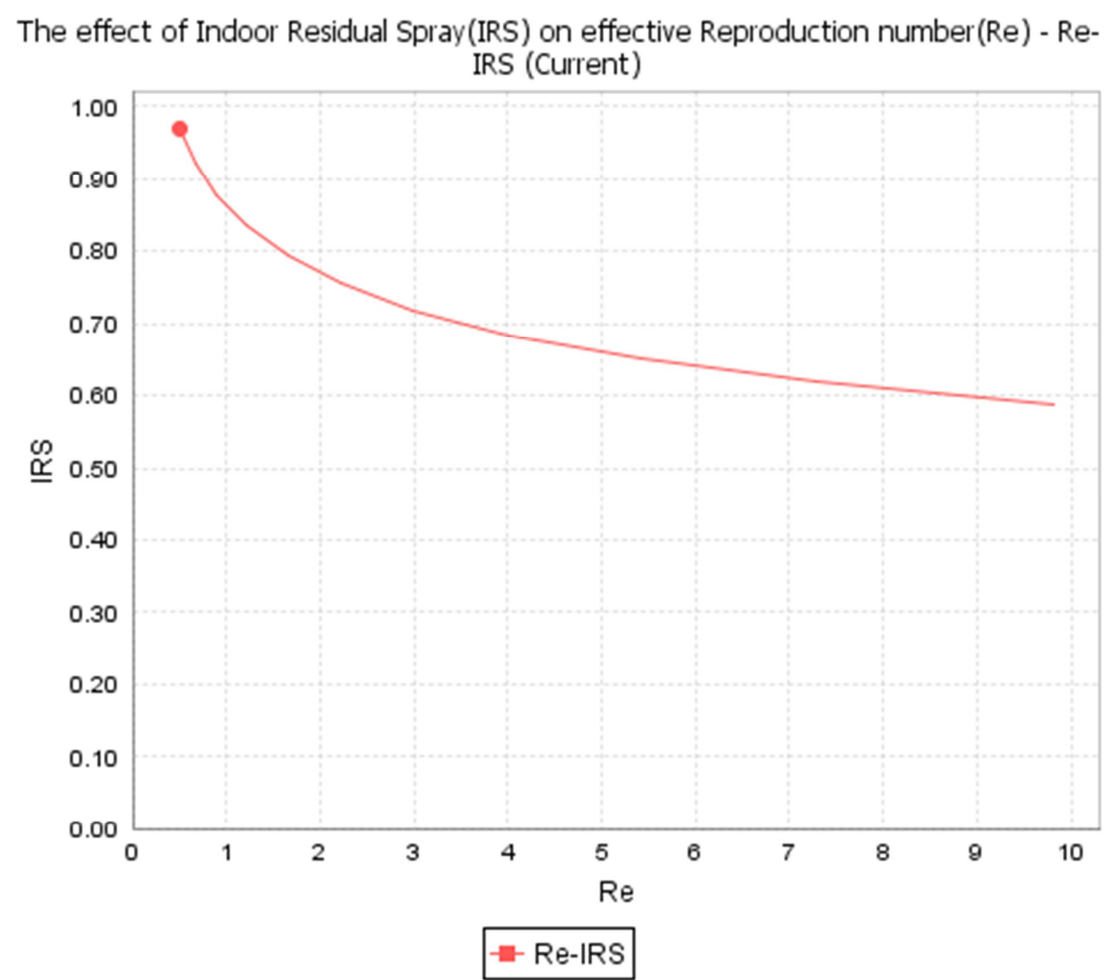

Figure 4. Indoor Residual SprayIRS VS Effective Reproduction number $R_{e}$ graph.

These plots show the effect of IRS on effective reproduction number, $R_{e}$ as a function of IRSand where IRS $=0.97$. Notice that the reproductive number drops below one for IRSbetween 0.85 and 1 .

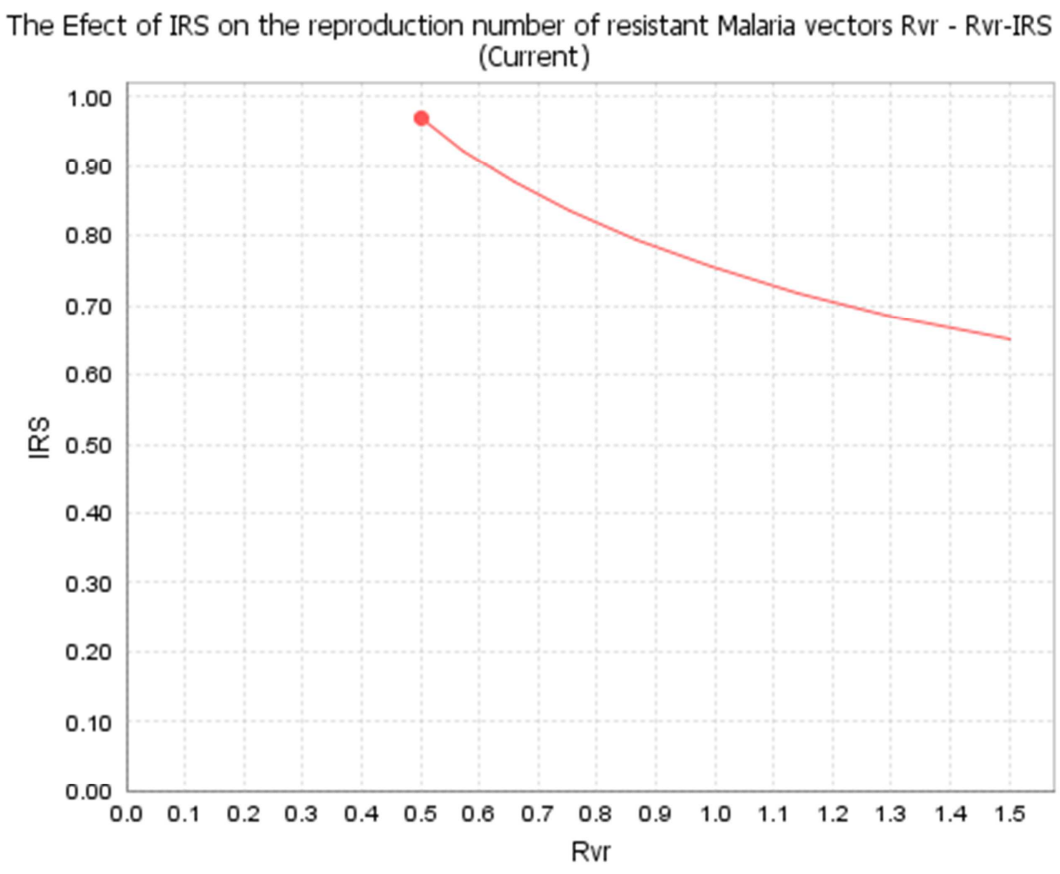

RVr-IRS

Figure 5. Indoor Residual SprayIRS VS Reproduction number for the Resistant malaria vector strains $R_{v r} g r a p h$. 
Figure 5 shows that the effect of IRSonthe reproduction numberofinsecticidesresistant malaria vector strains, $R_{v r}$ as a function of IRSandwhere IRS=0.97. Notice that the reproductive number drops below one for IRSbetween 0.75 and 1 .

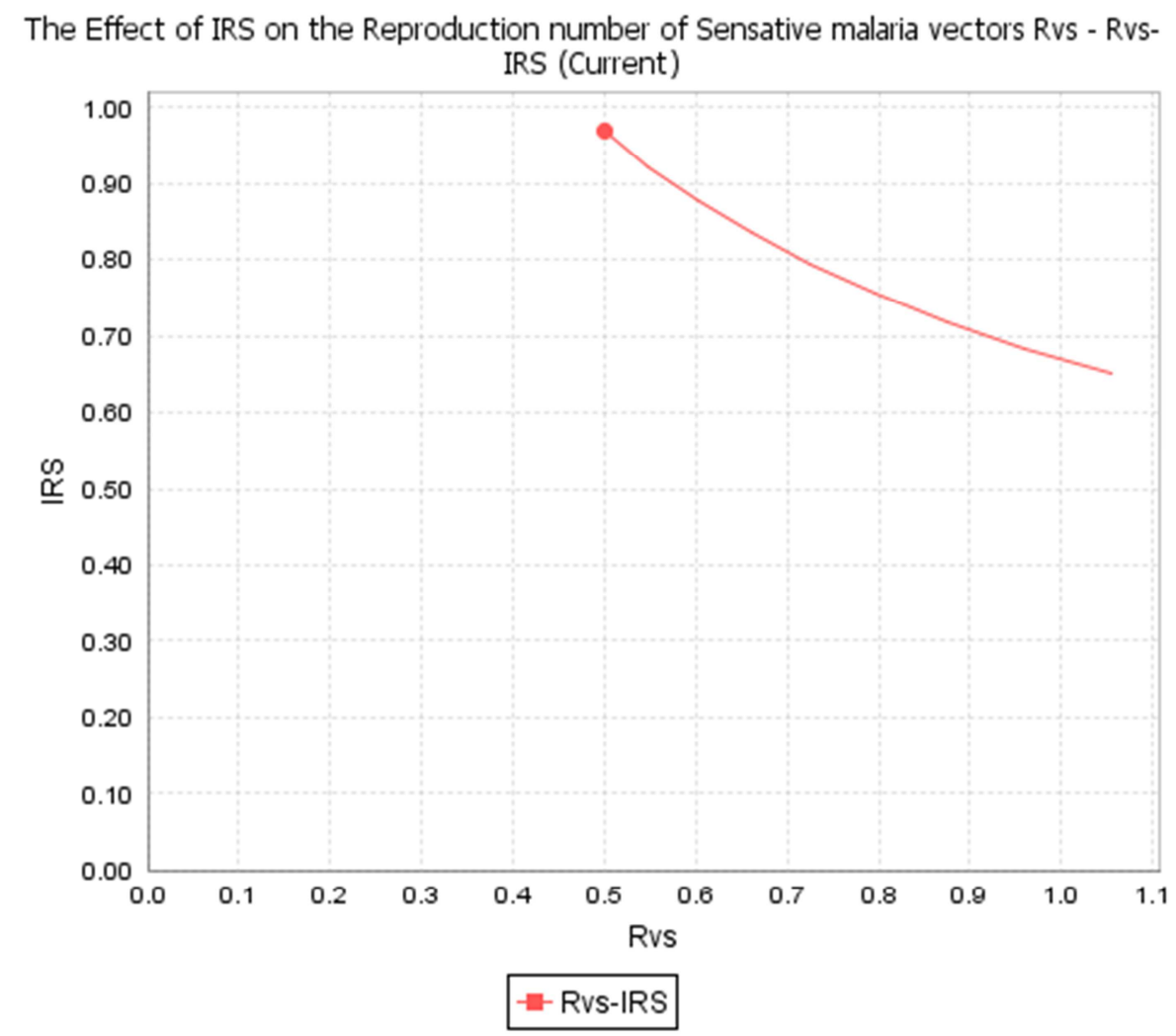

Figure 6. Indoor Residual SprayIRS VS Reproduction number for the sensitive malaria vectorR ${ }_{\text {vs }}$ graph.

Figure 6 shows that the effect of IRS on the reproductive number of insecticide sensitive malaria vector strains, $R_{v s}$ as a function of IRSand where IRS $=0.97$. Notice that the reproductive number drops below one for IRS between 0.65 and 1.

\section{Conclusion and Recommendation}

In our analysis of the modeling the dynamics of endemic malaria transmission with the effects of control measure (IRS), we ran a number of simulations using the initial population sizes and a set of parameter values in Table 3 and Table 4 respectively in this model. Where IRS, is ranging from 0 to 1 . We have seen that as the use IRS increases in amount, the reproductive number gets closer to 1 , eventually falling below that critical value. As the evolution of insecticide resistance that allows for small proportion of mosquitoes possessing resistance genes allowing them to resist and survive the effects of the insecticide (IRS) increases, the change in reproduction number of resistant malaria vector strains $R_{v r}$ against insecticide is clearly seen. This consequently resulted in increasing the overall effective reproduction number $R_{e}$. Because of only single insecticide (IRS) is used during intervention and (IRS) is not properly used at a time i.e., the same control measure is used for long periods without followed by rotation of different types of control measures, insecticide sensitive malaria vector strains progress to the insecticide resistant malaria vector strains. This also leads to an increase in reproduction number of insecticide resistant malaria vector strains $R_{v r}$, consequently increasing the overall effective reproduction number $R_{e}$ which is resulted in control intervention failure which also resulted in malaria disease burden in the community.

The public health implications of the results include: (i) every effort should be taken to minimize the evolution of insecticide resistance due to malaria control interventions failure and (ii) ) at least the combination of two different types of control measures and followed by rotation of intervention strategies could be more realistic to minimize the number of resistant malaria vector strains and essential in reducing the malaria disease burden in the community.

\section{Acknowledgements}

First of all would like to say thanks to my God who handed to me his peace. Next I would like to express my sincere gratitude and appreciation goes to my first adviser Dr. Purnachandra Rao Koya (Prof.) for his commitment to support me to do this work.

\section{References}

[1] Centers for Disease Control and Prevention. $C D C$ - Malaria (accessed August 8, 2011) http://www.cdc.gov/MALARIA. 
[2] Worrall E, Basu S, Hanson K. Is malaria a disease of poverty? A review of the literature. Tropical Medicine \& International Health.2005;10:1047-1059

[3] Hawass Z, Gad YZ, Ismail S, Khairat R, Fathalla D, Hasan N, et al. Ancestry and pathology in King Tutankhamun's family. JAMA. 2010; 303: 638-647. DOI: 10.1001/jama. 2010.121

[4] WHO. World Malaria Report 2017. Geneva, Switzerland: World Health Organization; 2017

[5] Bhatt S, Weiss D, Cameron E, Bisanzio D, Mappin B, Dalrymple U, et al. The effect of malaria control on Plasmodium falciparum in Africa between 2000 and 2015. Nature. 2015; 526:207-211

[6] WHO. Global Plan for Insecticide Resistance Management in Malaria Vectors. Geneva, Switzerland: World Health Organization; 2012. 132 pp. Available from: www.who.int/malaria/vector_control/ivm/gpirm/en/

[7] Davidson G. Insecticide resistance in Anopheles sundaicus. Nature. 1957;4598:1333-1335

[8] Gilbert LI, Gill SS. Insect Control: Biological and Synthetic Agents. London, United Kingdom: Academic Press; 2010. 470 $\mathrm{p}$

[9] Ross R. "The Prevention of Malaria”, John Murray, 1911.

[10] Macdonald G., "The Epidemiology and Control of Malaria", Oxford university press, 1957.

[11] J. L. Aron and R. M. May. The population dynamics of malaria. In The Population Dynamics of Infectious Diseases: Theory and Applications, pages 139-179. Springer, 1982.

[12] S. Gupta, J. Swinton, and R. M. Anderson. Proceedings of the Royal Society of London. Series B: Biological Sciences, 256(1347):231-238, 1994.

[13] J. C. Koella. Acta Tropica, 49(1):1-25, 1991.

[14] G. Macdonald. Proceedings of the Royal Society of Medicine, 48(4):295-302, 1955.

[15] G. Macdonald. The Epidemiology and Control of Malaria. Oxford University Press, 1957

[16] S. Ruan, D. Xiao, and J. C. Beier. Bulletin of Mathematical Biology, 70(4):1098-1114, 2008.

[17] D. L. Smith, K. E. Battle, S. I. Hay, C. M. Barker, T. W. Scott, and F. E. McKenzie. PLoS Pathogens, 8(4):e1002588, 2012

[18] Aron J. L., "Acquired immunity dependent upon exposure in a SIRS epidemic model", Journal ofMathematical Biosciences, vol. 88 , pp. 37-47, 1988.

[19] Aron J. L., "Mathematical modeling of immunity to Malaria", Journal of Mathematical Bio- sciences, vol. 90, pp. 385-396, 1988.
[20] Tumwiine J., Mugisha J., Luboobi L., "A mathematical model for the dynamics of malaria in a human host and mosquito vector with temporary immunity", Journal of Applied Mathematics and Computation, vol. 189, pp. 1953-1965, 2005.

[21] Tumwiine J., L. S. Luboobi, J. Y. T. Mugisha, "Modeling the effect of treatment and mosquitoes' control on malaria transmission", International Journal of Management and Systems, vol. 21, pp. 107-124, 2005.

[22] Yang H., Wei H., Li X., "Global stability of an epidemic model for vector borne disease", J Syst Sci Complex Journal, vol. 23, pp. 279-292, 2010.

[23] Fekadu Tadege Kobe \& Purnachandra Rao Koya, 'Spread of Malaria Disease Using Intervention Strategies 'Journal of multi disciplinary Engineering Science and Technology (JMEST), vol.2, Issse5, May-2015.

[24] E. C. Ibezim and U. odo, Current trends in malarial chemotherapy, African Journal of Biotechnology, 7 No.4 (2008), 349-356, 2014.

[25] O. Diekmann, J. A. P. Heesterbeek and J. A. J. Metz, on the definition and computation of the basic reproduction ratio in models for infectious diseases in heterogeneous populations, J. Math. Biol, 28 (1990), 365-382, doi: 10.1007/BF00178324.156 S.

[26] P. van den Driessche and J. Watmough, "Reproduction numbers and sub-threshold endemic equilibria for compartmental models of disease transmission," Mathematical Biosciences, vol. 180, pp. 29-48, 2002.

[27] J. C. Koella and R. Antia, "Epidemiological models for the spread of anti-malarial resistance," Malaria Journal, vol. 2, pp. $1-11,2003$

[28] H. Khalil. Nonlinear Systems, Prentice Hall, 2002.

[29] Merkin DR. Introduction to the theory of stability. SpringerVerlag New York, Inc; 1997.

[30] Chiyaka C., Tchuenche J. M., Garira W., and Dube S. A mathematical analysis of the effects of control strategies on the transmission dynamics of malaria. Appl. Math. Comput., 195(2):641 - 662, 2008.

[31] Chiyaka C., Garira W., and Dube S. Transmission model of endemic human malaria in a partially immune population. Math. Comput. Modelling, 46(5-6):806 - 822, 2007.

[32] Rowland M. Activity and mating competitiveness of gamma $\mathrm{HCH} /$ dieldrin resistant and susceptible male and virgin female Anopheles gambiae and An. stephensi mosquitoes, with assessment of an insecticide-rotation strategy. Medical and Veterinary Entomology. 1991; 5:207-222. 